based on: (i) the percentage of bone marrow blasts, including promonocytes, and (ii) the white blood cell count in the circulation. The latter criterion, though, is artificial, since most CMML cases present with a white blood cell count of $8-14 \times 10^{9} / \mathrm{L}$, often oscillating around the cut-off value of $13 \times 10^{9} / \mathrm{L}$.

All types of CMML show similarities with certain types of MDS. The paper by Valent et al. refers to a close relationship between MDS with multilineage dysplasia and dysplastic CMML-0, MDS with excess blasts-1 and dysplastic CMML-1, as well as MDS with excess blasts-2 and dysplastic CMML-2. In Figure 1, we try to illustrate the relationships within the large family of monocytoses.

Valent et al. propose the term "oligomonocytic CMML" in order to emphasize, as also pointed out by others, that it may be appropriate to diagnose CMML based on bone marrow monocytosis and CMML-typical somatic mutations, even if marked monocytosis is missing in the peripheral blood. ${ }^{12,13}$

The group also points out that CMML can display two different types of acceleration and progression. On the one hand, dysplastic CMML can adopt typical features of MPN by showing accelerated proliferation with organomegaly and constitutional symptoms, without necessarily producing an excess of blasts. This evolution from a dysplastic to a proliferative type may be heralded by increasing white cell counts, often accompanied by splenic enlargement. On the other hand, progression of CMML in terms of increasing blast percentage, i.e. from CMML-0 to CMML-1 or CMML-2, often occurs without a marked increase in white cell count or development of organomegaly. To complicate matters, both types of evolution can occur simultaneously or sequentially, and can be caused (or at least accompanied) by clonal evolution in terms of new somatic mutations, increased variant allele frequencies, or acquisition of chromosomal aberrations. As disease evolution may be more prominent in the bone marrow or peripheral blood, the authors recommend classifying the disease according to the highest blast count detectable.

Finally, the authors recommend complementing the group of CMML entities by including rare MPN-MDS variants, namely CMML with KITD816 $\mathrm{V}^{+}$systemic mastocytosis, and MDS-MPN with PDGFRA/B, FGFR1, or PCM-JAK2 rearrangements, because these rare entities are often associated with pronounced monocytosis.
The paper by Valent et al. provides a comprehensive description of all the diagnostic tools needed to assign patients to the appropriate category within the heterogeneous group of non-clonal and clonal monocytoses. It summarizes our current knowledge and represents a starting point for future refinements of the classification of bone marrow disorders characterized by monocytosis.

\section{References}

1. Valent P, Orazi A, Savona MR, et al. PProposed diagnostic criteria for classical chronic myelomonocytic leukemia (CMML), CMML variants and pre-CMML conditions. Haematologica. 2019;10410):1935-1949.

2. Bennett JM, Catovsky D, Daniel MT, et al. Proposals for the classification of the myelodysplastic syndromes. Br J Haematol. 1982;51(2):189199.

3. Brunning RD, Bennett JM, Flandrin G, et al. Myelodysplastic syndromes: introduction. In: Jaffe ES, Harris NL, Stein H, Vardiman JW editors. WHO Classification of Tumours of Haematopoietic and Lymphoid Tissues. 3rd ed. Lyon: IARC; 2001.

4. Orazi A, Bennett JM, Germing U, Brunning RD, Bain BJ, Thiele J. Chronic myelomonocytic leukemia. In: Swerdlow SH, Campo E, Harris NL, Jaffe ES, Pileri SA, Stein $\mathrm{H}$, et al., editors. WHO Classification of Tumours of Haematopoietic and Lymphoid Tissues. Lyon: IARC; 2008.

5. Orazi A, Bennett JM, Germing U, et al. Chronic myelomonocytic leukemia. In: Swerdlow SH, Campo E, Harris NL, Jaffe ES, Pileri SA, Stein $\mathrm{H}$, et al., editors. WHO Classification of Tumours of Haematopoietic and Lymphoid Tissues. Lyon: IARC; 2017.

6. Bowen DT. Chronic myelomonocytic leukemia: lost in classification? Hematol Oncol. 2005;23(1):26-33

7. Jaiswal S, Fontanillas P, Flannick J, et al. Age-related clonal hematopoiesis associated with adverse outcomes. N Engl J Med. 2014;371(26):2488-2498.

8. Steensma DP, Bejar R, Jaiswal S, et al. Clonal hematopoiesis of indeterminate potential and its distinction from myelodysplastic syndromes. Blood. 2015;126(1):9-16.

9. Valent P, Kern W, Hoermann G, et al. Clonal hematopoiesis with oncogenic potential (CHOP): separation from CHIP and roads to AML. Int J Mol Sci. 2019;20(3).

10. Cargo C, Cullen M, Taylor J, et al. The use of targeted sequencing and flow cytometry to identify patients with a clinically significant monocytosis. Blood. 2019;133(12):1325-1334.

11. Cazzola M. Clonal monocytosis of clinical significance. Blood. 2019;133(12):1271-1272.

12. Geyer JT, Tam W, Liu YC, et al. Oligomonocytic chronic myelomonocytic leukemia (chronic myelomonocytic leukemia without absolute monocytosis) displays a similar clinicopathologic and mutational profile to classical chronic myelomonocytic leukemia. Mod Pathol. 2017;30(9):1213-1222.

13. Schuler E, Frank F, Hildebrandt B, et al. Myelodysplastic syndromes without peripheral monocytosis but with evidence of marrow monocytosis share clinical and molecular characteristics with CMML. Leuk Res. 2018;65:1-4.

\title{
p66Shc deficiency sets the scene for clinically aggressive chronic lymphocytic leukemia
}

\section{Richard Rosenquist}

\author{
Dept. of Molecular Medicine and Surgery, Karolinska Institutet, Stockholm, Sweden \\ E-mail: RICHARD ROSENQUIST - richard.rosenquist@ki.se
}

doi:10.3324/haematol.2019.225904

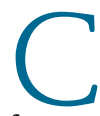
hronic lymphocytic leukemia (CLL) is a paradigmatic malignancy in which both cell-extrinsic (microenvironmental) and cell-intrinsic (genetic) factors contribute not only to the pathogenesis of the disease but also to disease evolution and outcome. ${ }^{1,2}$ In more recent years, the genomic landscape of CLL has been unraveled with the identification of "driver" gene muta- tions associated with clinical aggressiveness and chemorefractory disease, such as ATM, BIRC3, NOTCH1, NFKBIE, SF3B 1 and TP 53..$^{3-5}$ In addition to genetic aberrations, we also know that the $\mathrm{B}$-cell receptor (BCR) immunoglobulin plays a pivotal role in driving the disease onset and evolution. The somatic hypermutation status of IGHV genes divides patients into two major 
clinical subgroups of CLL, with IGHV-unmutated patients displaying a more rapidly progressing disease and an overall poor survival compared with IGHV-mutated patients. ${ }^{6,7}$ The importance of BCR signaling was reinforced with the successful introduction of BCR inhibitors (e.g. ibrutinib) in the treatment of CLL, which abrogate downstream BCR signaling and are effective in patients with a poor prognosis, i.e. patients with TP53 aberrations and/or unmutated IGHV genes. ${ }^{8}$ Finally, the micromilieau strongly contributes to CLL cell survival through direct/indirect interactions with different cell types (e.g., $\mathrm{T}$ cells, stromal cells) and receptors (e.g. CRCX4 and CCR7), in particular within the proliferation centers in secondary lymphoid organs.?

One of the few existing in vivo CLL models is the transgenic E $\mu$-TCL1 mouse, which recapitulates clinically aggressive human CLL. ${ }^{10}$ Thanks to this model, it is possible to study the different phases of disease development. In the current issue of Haematologica, Petrussi et al. have taken advantage of the E $\mu$-TCL1 mouse model to investigate the potential role of p66Shc deficiency in CLL pathobiology. ${ }^{11}$ The authors previously reported a significant association between p66Shc deficiency and dismal outcome, potentially linking this event to clinical aggressiveness. ${ }^{12}$ p66Shc is a Shc family adaptor that promotes production of reactive oxygen species (ROS), which in turn activate cell apoptosis. p66Shc is also known to act as a negative regulator of BCR signaling and to regulate lymphocyte homing by controlling the expression of different chemokine receptors (e.g., CXCR4 and CCR7). ${ }^{13}$

In their present study, Patrussi and colleagues first showed that leukemic B cells from E $\mu$-TCL1 mice with more advanced disease displayed lower levels of p66Shc expression compared with the levels in normal B cells. ${ }^{11}$ STAT4, an essential transcription factor for p66Shc, ${ }^{14}$ was also downregulated in E $\mu$-TCL1 mice. Notably, by treating leukemic E $\mu$-TCL1 splenic cells with ibrutinib they could restore p66Shc expression along with STAT4 expression, similar to what they had reported earlier in primary CLL. ${ }^{13}$

Next, by crossing the E $\mu$-TCL1 mice with p66Shc deficient mice, to produce $\mathrm{E} \mu$-TCL1/p66Shc/ mice, the latter demonstrated a markedly more rapid increase of $\mathrm{CD}^{+} / \mathrm{CD} 19^{+}$cells in peripheral blood, a 2 -month earlier disease onset and shorter overall survival compared to $\mathrm{E} \mu$-TCL1 mice. ${ }^{11}$ When leukemic cells from $\mathrm{E} \mu$ TCL1/p66Shc/. mice, which expressed higher Bcl2 levels, were treated with fludarabine, they were more resistant than $\mathrm{E} \mu$-TCL1 cells, indicating that p66Shc loss also contributes to decreased chemo-sensitivity.

The E $\mu$-TCL1 model also offers the unique possibility to study the pattern of disease infiltration. In $\mathrm{E} \mu$ TCL1/p66Shc mice, an increased accumulation of leukemic cells was observed in both nodal and extranodal sites; higher percentages and proliferation rates of leukemic cells were preferentially seen in lymph nodes, liver and lung, but not in spleen and bone marrow. While expression of CXCR4 was unchanged in leukemic cells, the expression of CCR7, a key lymph node B-cell homing receptor, CCR2 (associated with both lung and liver homing) and CXCR3 (linked to lung homing) was higher in $\mathrm{E} \mu$-TCL1/p66Shc- leukemic cells than in cells from $\mathrm{E} \mu$ -
TCL1 mice. ${ }^{11}$ Hence, a more efficient accumulation in nodal and extranodal sites appears to depend on distorted chemokine receptor expression due to p66Shc deficiency.

p66Shc deficiency was also confirmed in primary CLL cells, in particular in IGHV-unmutated CLL; however, the authors could not find any correlation with 13q deletion or TP53 aberrations. Similar to E $\mu$-TCL1/p66Shc mice, both CCR2 and CXCR3 were overexpressed in IGHVunmutated CLL, and p66Shc re-expression in CLL cells resulted in decreases in CCR2 and CXCR3 mRNA expression. The number and size of infiltrated lymph nodes and presence of spleen and/or liver enlargement were also higher in patients with low p66Shc mRNA levels. Interestingly, the authors observed that p66Shc expression was higher in CLL patients with a known response to second-line ibrutinib treatment than in patients who did not respond.

Finally, Patrussi et al. demonstrated a correlation between decreased ROS production and p66Shc deficiency both in CLL cells and E $\mu$-TCL1/p66Shc ${ }^{-/}$cells. ${ }^{11}$ Using transfection experiments, wildtype p66Shc-expressing transfectants, but not the ROS-defective mutant, showed lower CCR2 and CXCR3 expression compared to control cells, implying that p66Shc directly modulates CCR2 and CXCR3 expression through elevation of ROS.

Based on the novel results from this study, p66Shc deficiency in E $\mu$-TCL1 mice was shown to accelerate disease onset and progression to an aggressive phenotype. The authors also followed organ selectivity which correlated with deregulation of specific chemokine receptors. Importantly, p66Shc expression could be restored by ibrutinib treatment, along with enhanced chemo-sensitivity. Considering the potential role of p66Shc deficiency preceding a more aggressive phase of the disease, it will now be important to follow CLL patients longitudinally and in relation to therapy in order to determine if p66Shc could be used as a potential biomarker. Could p66Shc expression predict a shift from a more indolent to a more aggressive disease in need of therapy? Could p66Shc be monitored after treatment with chemo-immunotherapy or targeted therapy as an early indicator of disease progression/chemo-refractoriness? From a biological point of view, it will also be important to identify what cellular/molecular factors are involved in and lead to p66Shc downregulation. Do other cell types in the CLL microenvironment influence p66Shc levels? Is there any correlation with gene mutations affecting key cellular pathways and processes linked to an aggressive phenotype or chemo-resistance? While the study by Petrussi et al. using E $\mu$-TCL1/p66Shc mice has provided us with important insights, further studies are now necessary to investigate the potential clinical role of p66Shc deficiency, preferably in uniformly treated cohorts and involving novel therapies.

\section{References}

1. Sutton LA, Rosenquist R. Deciphering the molecular landscape in chronic lymphocytic leukemia: time frame of disease evolution. Haematologica. 2015;100(1):7-16.

2. Sutton LA, Rosenquist R. The complex interplay between cell-intrinsic and cell-extrinsic factors driving the evolution of chronic lymphocytic leukemia. Semin Cancer Biol. 2015;34:22-35. 
3. Landau DA, Tausch E, Taylor-Weiner AN, et al. Mutations driving CLL and their evolution in progression and relapse. Nature. 2015;526(7574):525-530

4. Mansouri L, Sutton LA, Ljungstrom V, et al. Functional loss of IkappaBepsilon leads to NF-kappaB deregulation in aggressive chronic lymphocytic leukemia. J Exp Med. 2015;212(6):833-843.

5. Puente XS, Bea S, Valdes-Mas R, et al. Non-coding recurrent mutations in chronic lymphocytic leukaemia. Nature. 2015;526(7574):519-524

6. Rosenquist R, Ghia P, Hadzidimitriou A, et al. Immunoglobulin gene sequence analysis in chronic lymphocytic leukemia: updated ERIC recommendations. Leukemia. 2017;31(7):1477-1481.

7. Sutton LA, Hadzidimitriou A, Baliakas $\mathrm{P}$, et al. Immunoglobulin genes in chronic lymphocytic leukemia: key to understanding the disease and improving risk stratification. Haematologica. 2017;102(6):968-971

8. Byrd JC, O'Brien S, James DF. Ibrutinib in relapsed chronic lymphocytic leukemia. N Engl J Med. 2013;369(13):1278-1279.

9. Caligaris-Cappio F, Bertilaccio MT, Scielzo C. How the microenvironment wires the natural history of chronic lymphocytic leukemia.
Semin Cancer Biol. 2014;24:43-48.

10. Johnson AJ, Lucas DM, Muthusamy N, et al. Characterization of the TCL-1 transgenic mouse as a preclinical drug development tool for human chronic lymphocytic leukemia. Blood. 2006;108(4):13341338

11. Patrussi L, Capitani N, Ulivieri C, et al. p66Shc deficiency in the Emu-TCL1 mouse model of chronic lymphocytic leukemia enhances leukemogenesis by altering the chemokine receptor landscape. Haematologica. 2019;104(10):2040-2052.

12. Capitani N, Lucherini OM, Sozzi E, et al. Impaired expression of p66Shc, a novel regulator of B-cell survival, in chronic lymphocytic leukemia. Blood. 2010;115(18):3726-3736.

13. Patrussi L, Capitani N, Cattaneo F, et al. p66Shc deficiency enhance CXCR4 and CCR7 recycling in CLL B cells by facilitating their dephosphorylation-dependent release from beta-arrestin at early endosomes. Oncogene. 2018;37(11):1534-1550.

14. Cattaneo F, Patrussi L, Capitani N, et al. Expression of the p66Shc protein adaptor is regulated by the activator of transcription STAT4 in normal and chronic lymphocytic leukemia B cells. Oncotarget. 2016;7(35):57086-57098.

\title{
Hereditary thrombotic thrombocytopenic purpura
}

\author{
Marie Scully \\ Department of Haematology, UCLH and Cardiometabolic Programme-NIHR UCLH/UC BRC London, UK \\ E-mail: MARIE SCULLY - m.scully@nhs.net
}

doi:10.3324/haematol.2019.225896

T he first description of thrombotic thrombocytopenic purpura (TTP) by Moschowitz was published nearly 100 years ago. ${ }^{1}$ This was likely to have been an immune-mediated TTP episode and the author described multi organs affected with worsening, untreated disease. Accounts of hereditary TTP were otherwise acknowledged to be Upshaw Shulman syndrome. In 1960, Schulman reported an 8-year old girl who had repeated episodes of thrombocytopenia and hemolytic anemia from infancy. Treatment with plasma was associated with normalization of the platelet count and resolution of hemolysis, and remission was maintained with prophylactic plasma every 1-2 weeks. ${ }^{2}$ Upshaw presented a 16-year old girl with relapsing hemolytic anemia and thrombocytopenia since infancy. The patient responded to blood transfusions. During the next 11 years, Upshaw treated 32 episodes of thrombocytopenia and microangiopathic hemolysis with plasma infusions. The acute episodes invariably had a trigger, such as a minor infection, surgical procedure, pregnancy, or pancreatitis. Acute intervals lasted from three weeks to 20 months, at which time the platelet count was normal and there was a compensated hemolysis. Between these acute episodes, it was observed that intravascular platelet and red cell survival was shortened; these abnormalities normalized after the infusion of two units of plasma. ${ }^{3}$

In this edition of Haematologica, Van Dorland et al. ${ }^{4}$ present an international collaborative study on hereditary TTP. As an ultra-rare disorder, collection of meaningful data is critical to understand the clinical features of this condition, the therapy, and the long-term impact. The international registry presents data from over an 11-year period, incorporating 123 patients from four continents who presented the disease from the neonatal period, up to the seventh decade of life. We know, from numerous publications relating to the mutations identified in hereditary TTP, that there is a heterogenous distribution throughout the ADAMTS 13 gene. ${ }^{5-8}$ There are, however, two specific variants that have been identified at increased frequency in hereditary TTP.
R1060W, exon 24, is prominent in Caucasians presenting with late onset congenital TTP specifically associated with pregnancy. ${ }^{9,10}$ Within the international hereditary TTP registry, c.4143_4144dupA (exon 29; p.Glu1382Argfs*6) was prevalent, specifically within northern Europe, and was initially described at increased prevalence from central Norway. ${ }^{11}$ Further cases were documented in the international registry, confirming that those patients with compound heterozygous mutations were more likely to have an earlier presentation, specifically in the neonatal period. There were other mutations which have been identified in more than one individual in the international registry. This allows us to predict the impact of such variations within the ADAMTS 13 gene with respect to clinical features. Such observations are only possible within the breadth of a registry. ${ }^{4}$

The international registry has identified the significant delay in diagnosis of hereditary TTP; overall median age of overt presentation was 4.5 years but the clinical diagnosis was not made until a median of 16.7 years. Acute TTP episodes occurred at a median rate of 0.1 per year, ranging up to nine per year, and triggers included infections, childbirth, trauma, and in males, excess alcohol intake. The median time to resolution following an acute presentation was seven days.

There was an equal male to female ratio; this is in contrast to immune-mediated disease, which has a female preponderance. Residual ADAMTS 13 activity could not reliably predict age of onset or, indeed, disease severity, despite a cut-off of $1 \%$. This may also help to explain why patients with identical genetic variants have significant differences in the clinical phenotype. What is particularly striking and important is the clinical impact of hereditary TTP: 1) the degree of end-organ damage symptoms, either at presentation or as complications of the disease; and 2) the proportion of patients with arterial thromboembolic events, present in 28\% and covering all age groups. This was especially striking in $40-50$ years old; $>50 \%$ of these patients had at least one arterial thromboembolic event. 